\title{
Electric field effects in Fibonacci superlattices
}

\author{
Mario Castro ${ }^{\dagger}$ and Francisco Domínguez-Adame ${ }^{\ddagger}$ \\ Departamento de Física de Materiales, Facultad de Físicas, Universidad Complutense, E-28040 Madrid, Spain \\ and \\ Grupo Interdisciplinar de Sistemas Complicados, Escuela Politécnica Superior, Universidad Carlos III, E-28911 Leganés, \\ Madrid, Spain
}

\begin{abstract}
We present a throughout study of transmission and localization properties of Fibonacci superlattices, both in flat band conditions and subject to homogeneous electric fields perpendicular to the layers. We use the transfer matrix formalism to determine the transmission coefficient and the degree of localization of the electronic states. We find that the fragmentation pattern of the electronic spectrum is strongly modified when the electric field is switched on, this effect being more noticeable as the system length increases. We relate those phenomena to field-induced localization of carriers in Fibonacci superlattices.
\end{abstract}

PACS numbers: 71.50.+t, 72.15.Rn, 73.20.Dx

\section{INTRODUCTION}

The fabrication of aperiodic semiconductor superlattices (SLs), arranged according to the Fibonacci [1] and Thue-Morse [2] sequences, has given rise to a growing interest in their exotic electronic and transport properties [3 12]. Theoretical studies demonstrate that ideal aperiodic SLs should exhibit a highly-fragmented and fractal-like electronic spectrum [1,9611]. This self-similar spectrum is observable even when unintentional imperfections arising during the growth process are considered [12]. The electronic states associated to this peculiar spectrum are no longer Bloch states and also present fractal-like properties [13, 14, although they extend over the entire sample, what is most important for subsequent applications in actual devices, these novel properties have been experimentally observed. For instance, photoluminescence excitation spectroscopy at low temperature reveals the existence of a fragmented density of states consistent with theoretical predictions $[5]$.

Most devices work under bias conditions and, consequently, a complete characterization of electronic states in aperiodic SLs subject to an applied electric field is indeed needed. In this way, it has been recently demonstrated that time-dependent coherent oscillations of electronic wavepackets induced by the homogeneous electric field (Bloch oscillations) are absent in these SLs [15]. This is to be compared with periodic SLs, where Bloch oscillations have been predicted and detected in $\mathrm{Ga}_{1-x} \mathrm{Al}_{x}$ As [16, 17]. In this paper we address the study of the energy spectrum and transmission property of Fibonacci SLs (FSLs) subject to an applied electric field. We aim to get deep insight into the interaction of fractallike electronic states of FLSs with the external field, which clearly is more complex than in the case of periodic SLs. We will focus our attention on the effects of this electric field on the fragmented spectrum. Moreover, we will also discuss the competition between the longrange order of the FSL and the localization effects of the external electric field. To be specific, we consider the scattering problem of an electron impinging on a quasione dimensional FSL. Transport properties for different electron energies will be described by means of the transmission coefficient since this magnitude is directly related to the conductance of the sample. To get an estimation of the degree of localization of the electronic state as a function of energy, we will use the inverse participation ratio (IPR) to be defined below. These two magnitudes will be shown below to be enough for our present purposes.

\section{MODEL}

We consider quantum-well based SLs with the same barrier thickness $b$ in the whole sample. The height of the $n$th barrier with center at $z_{n}=n a$ barrier is given by the conduction-band offset, $z$ being the coordinate in the growth direction and $a>b$ the separation between neighbouring barriers. We will focus on electronic states close to the bandgap with $\mathbf{k}_{\perp}=0$ and neglect nonparabolicity effects hereafter, so that the Ben DanielDuke Hamiltonian suffices to describe those states. For the sake of simplicity, we consider FSLs with narrow barriers, namely we assume strong coupling between quantum wells. From a mathematical point of view we require that $b \rightarrow 0$ whereas the area of the barrier remain unchanged ( $\delta$-function limit). Therefore, the envelopefunctions for electronic states satisfy the following timeindependent Schrödinger equation

$$
\left[-\frac{\hbar^{2}}{2 m} \frac{d^{2}}{d z^{2}}+\sum_{n=1}^{N} V_{n} a \delta(z-n a)-e F z\right] \psi(z)=E \psi(z),
$$

where $V_{n} a$ is the strength of the $\delta$-function, $N$ is the number of barriers and $F$ is the electric field. We take the origin of electron energies at the conduction-band edge in the quantum-wells. FSLs can be grown starting from 
two different barriers with strengths $V$ and $V^{\prime}$, arranged according to the Fibonacci sequence. The Fibonacci sequence $S_{n}$ is generated by appending the $n-2$ sequence to the $n-1$ one, i.e., $S_{n}=S_{n-1} S_{n-2}$, where $S_{0}=V^{\prime}$ and $S_{1}=V$. Thus, finite and self-similar quasiperiodic SLs are obtained by $n$ successive applications of these rules containing $N=F_{n}$ barriers arranged according $V V^{\prime} V V V^{\prime} \ldots$ The Fibonacci numbers are generated from the recurrence law $F_{n}=F_{n-1}+F_{n-2}$, starting with $F_{0}=F_{1}=1$.

To proceed, we use the transfer-matrix method to calculate the transmission coefficient, in a similar fashion to the case of periodic SLs [18]. We define the length $\ell=\ell(F) \equiv\left(\hbar^{2} / 2 m e F\right)^{1 / 3}$, the dimensionless parameter and $\lambda=\lambda(F, E) \equiv\left(2 m / \hbar^{2} e^{2} F^{2}\right)^{1 / 3} E$, and the dimensionless variable $y=y(z, F, E) \equiv(2 / 3)[\lambda+z / \ell]^{3 / 2}$, in order to obtain the solution of (11) in terms of the Hankel functions of first and second kind

$\psi_{n}(z)=A_{n} y^{1 / 3} H_{1 / 3}^{(1)}(y)+B_{n} y^{1 / 3} H_{1 / 3}^{(2)}(y), \quad x_{n-1}<x<x_{n}$,

where the constants $A_{n}$ and $B_{n}$ are to be determined from the boundary conditions. The coefficients in the free-field regions $\left(x<x_{0}\right.$ and $\left.x>x_{N}\right)$ are related through the transfer matrix $T_{N}$ as follows:

$$
\left(\begin{array}{l}
A_{N} \\
B_{N}
\end{array}\right)=T_{N}\left(\begin{array}{c}
A_{0} \\
B_{0}
\end{array}\right) \equiv \prod_{n=N}^{1} M_{n}\left(\begin{array}{c}
A_{0} \\
B_{0}
\end{array}\right)
$$

where

$$
M_{n}=\left(\begin{array}{cc}
1+\alpha_{n} f_{n} / i_{n} & \alpha_{n} g_{n} / i_{n} \\
-\alpha_{n} h_{n} / i_{n} & 1-\alpha_{n} f_{n} / i_{n}
\end{array}\right)
$$

is the site transfer matrix and for brevity we have defined $\alpha_{n}=\left(2 m \ell V_{n} a / \hbar^{2}\right)\left(3 y_{n} / 2\right)^{-1 / 3}$ with $y_{n}=$ $y(n a, F, E), f_{n}=H_{1 / 3}^{(2)}(y) H_{-2 / 3}^{(1)}(y)-H_{1 / 3}^{(1)}(y) H_{-2 / 3}^{(2)}(y)$, $g_{n}=H_{1 / 3}^{(2)}(y) H_{1 / 3}^{(1)}(y), h_{n}=\left[H_{1 / 3}^{(2)}(y)\right]^{(2)}$ and $i_{n}=$ $\left[H_{1 / 3}^{(1)}(y)\right]^{(2)}$. The total transfer matrix $\mathcal{T}_{N}$ relates the amplitudes of plane waves in the free-field regions and the transmission coefficient is then determined from the relationship $\tau=\left|\operatorname{det}\left(\mathcal{T}_{N}\right) /\left(\mathcal{T}_{N}\right)_{22}\right|^{2}$.

As mentioned in the Introduction, we use the IPR to determine the degree of localization of the wave function for different incoming energies. The amplitude distribution of the electronic states can be characterized by the moments associated to the measure defined in the system by us (in our case the probability of finding the electron at a given point of the lattice). We then use the second moment of this distribution, which is nothing but the so-called IPR, defined as follows

$$
\operatorname{IPR}=\frac{\sum_{n=1}^{N}\left|\psi_{n}(n a)\right|^{4}}{\left(\sum_{n=1}^{N}\left|\psi_{n}(n a)\right|^{2}\right)^{2}} .
$$

The IPR is usually used to evaluate the degree of localization of electronic states. Delocalized states are expected to present small IPR, of order of $N^{-1}$, while localized states have larger IPR.

\section{RESULTS AND DISCUSSIONS}

As typical values of physical parameters we take $V=$ $25 \mathrm{meV}, V^{\prime}=28 \mathrm{meV}$ and $a=10 \AA$. To simplify the numerical analysis and to facilitate direct comparison with previous results in periodic SLs [18], we define two dimensionless quantities. We then introduce the reduced en$\operatorname{ergy} \varepsilon=E / V$ and the reduced electric field $\tilde{F}=e F a / V$. In addition, we focus our attention in the first allowed miniband of the SL, which ranges from $\varepsilon=0.9$ to $\varepsilon=14.2$ in the periodic SL with our chosen parameters.

FIGURE 1

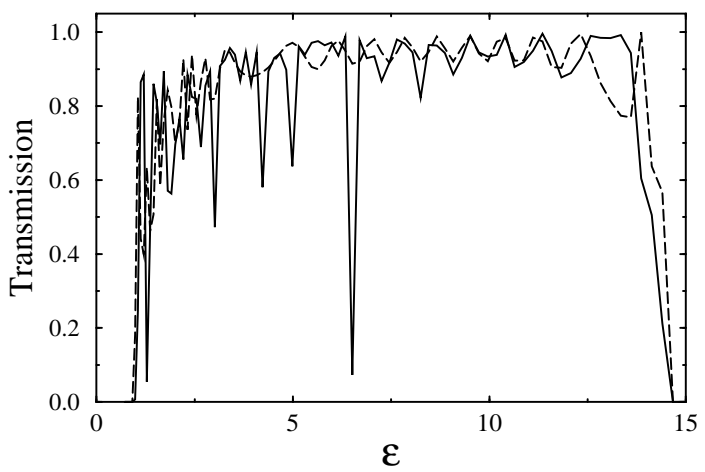

FIG. 1. Transmission coefficient as a function of the electron energy in periodic (dashed line) and Fibonacci superlattices (solid line) under flat band conditions. The number of barriers is $N=F_{13}=377$ in both cases.

Figure 1 collects our results on transmission in both both periodic and Fibonacci SLs with $N=F_{13}=377$ under flat band conditions. Results demonstrate that the miniband structure is changed when quasiperiodicity is introduced in the SL. Notice the occurrence of gaps within the allowed miniband, which are absent in the periodic SL. This phenomenon is by now well understood (see Ref. [9] and references therein), and is due to the reduction of the resonant coupling between neighbouring quantum wells. We should also mention that this picture does not change very much on increasing the SL length due to the so-called asymptotic stability [9], i. e. the global properties can be obtained in practice by considering very short approximants to the FSL, whereas increasing the system length leads to change only finer details. 

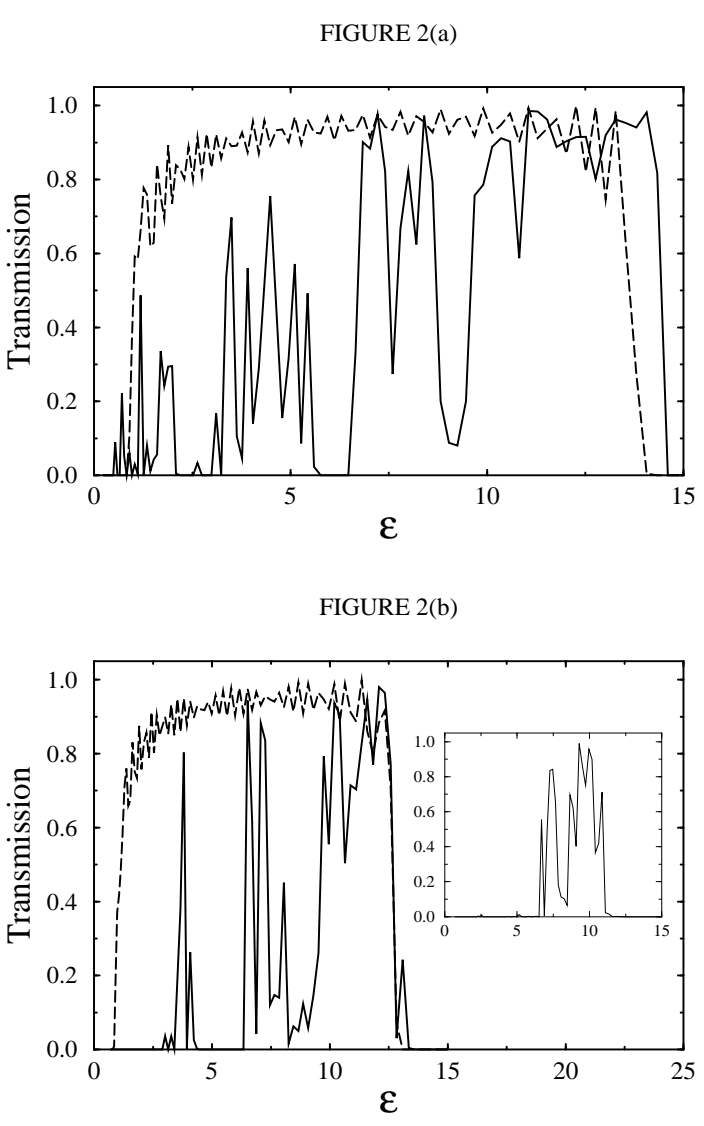

FIG. 2. Transmission coefficient as a function of the electron energy in periodic (dashed lines) and Fibonacci (solid lines) SLs with (a) $N=144$ and (b) $N=377$ barriers. The applied electric field is $\tilde{F}=0.005$ in all cases. Inset shows the transmission coefficient for the FSL with $N=F_{13}=377$ at $\tilde{F}=0.01$

The above scenario under flat band conditions is no longer valid when moderate electric fields are applied. Fig. 2 shows the results when $\tilde{F}=0.005$ for both periodic and Fibonacci SLs with different lengths. In the case of periodic SL, only minor differences can be detected on increasing the lenght [see Fig. 2(a) and (b)], besides a decrease of the upper miniband-edge. On the contrary, marked differences appear in the case of FSLs when increasing the length when an applied electric field is applied. By comparing Fig. 2(a) and (b), we observe a strong reduction of transmission properties, especially at the lower miniband-edge, where several transmission peaks completely disappear. Thus we are led to the conclusion that the interplay between the long-range order of FSLs and the localization properties of the electric field effects are more complex than in periodic SLs. These differences clearly arise from the very different nature of the eigenstates in both types of SLs 14. In particular, we deduce from the above results that the localization properties of the electric field are more pronounced in the case of FSLs. On increasing the electric field the miniband shrinks, as can be seen in the inset if Fig. 2(b), especially at low energies. Further increase of the electric filed leads to the vanishing of the miniband, as occurs in periodic SLs 18 .



FIG. 3. Ratio of the IPR in FSL and in periodic SL as a function of the electron energy with $N=F_{11}=144$ (dashed line) and $N=F_{13}=377$ (solid line) barriers. The applied electric field is $\tilde{F}=0.005$ in both cases.

To obtain further confirmation of the above statement we have studied the degree of localization of wave functions. In order to facilitate comparison between the localization under flat band and subject to an applied electric field, we define a new parameter $R$ as the ratio of the IPR of the FSL and the IPR of the periodic SL at the same values of the energy, electric field and system length. Results are collected in Fig. 3 for $N=F_{11}=144$. At flat band the value of the parameter $R$ fluctuates around unity, as it can be seen in Fig. 3. This implies that the degree of localization is similar in both kinds of SL. This agree with previous observations that electronic states spread over the entire Fibonacci system, although the nature of those states is completely different from those in periodic systems 14. However, as soon as the electric field is applied, electrons are much more localized in the FSL than in the periodic SL, as demonstate the dramatic increase of the parameter $R$ shown in Fig. 3. Notice that the peaks of $R$ correspond to the gaps revealed in the trasmission coefficient. This observation reinforces our claim that the localization effects of the electric field are stronger in FSL.

\section{CONCLUSION}

In this paper we have studied the transmission properties of electrons in Fibonacci semiconductor superlattices subject to an electric field. Results have been compared to those obtained in periodic superlattices with the same parameters. By taking the approximation of narrow barriers, which is of interest when resonant coupling between neigbouring barriers takes place and consequently minibands arise, we have been able to obtain a closed expres- 
sion of the trasmission coefficient within the transfer matrix formalism. In addition, the same approach allows us to discuss the degree of localization of wave functions by means of the inverse participation ratio. We have found that at flat band small gaps appear within the lower miniband as soon as quasiperiodicity is introduced in the sample. However, the Fibonacci SL still presents good transmission properties, which is consistent with the fact that electronic states spread over the entire sample in the absence of dc voltage. On the contrary, dramatic changes occur whenever the dc field is switched on. In particular, we have found a strong reduction of the transmission properties, this reduction being more significant as the length of the system increases. The IPR confirms these results and points out that electronic states are much more localized in Fibonacci SLs than in periodic ones under the same bias conditions. For large enough electric fileds one could expect that the fragmented pattern of the electronic spectrum will be severely modified. We believe that this result is very important from a practical point of view since it demonstrates that changes in the electronic structure should be taken into account when samples are driven by a dc field.

\section{ACKNOWLEDGMENTS}

The authors thank Enrique Maciá and Angel Sánchez for helpful comments. This work has been supported by CICYT (Spain) under project MAT95-0325.

$\dagger \quad$ Electronic address: mario@valbuena.fis.ucm.es

¥ Electronic address: adame@valbuena.fis.ucm.es

[1] R. Merlin, K. Bajema, R. Clarke, F. -Y. Juang, and P. Bhattacharya, Phys. Rev. Lett. 55, 1768 (1985).

[2] R. Merlin, K. Bajema, J. Nagle, and K. Ploog, J. Phys. (Paris) Colloq. 48, C5-503 (1987).

[3] R. Merlin, IEEE J. Quantum Electron. 24, 1791 (1988).

[4] F. Laruelle and B. Etienne, Phys. Rev. B 37, 4816 (1988).

[5] A. A. Yamaguchi, T. Saiki, T. Tada, T. Ninomiya, K. Misawa, and T. Kobayashi, ibid. 75, 955 (1990).

[6] D. Toet, M. Potemski, Y. Y. Wang, J. C. Maan, L. Tapfter, and K. Ploog, Phys. Rev. Lett. 66, 2128 (1991).

[7] K. Hirose, D. Y. K. Ko, and H. Kamimura, J. Phys.: Condens. Matter 4, 5947 (1992).

[8] D. Munzar, L. Bočáek, J. Humlíček, and K. Ploog, J. Phys.: Condens. Matter 6, 4107 (1994).

[9] E. Maciá, F. Domínguez-Adame, and A. Sánchez, Phys. Rev. B 49, 9503 (1994).

[10] F. Domínguez-Adame, E. Maciá, B. Méndez, Phys. Lett. A 194, 184 (1994).

[11] F. Domínguez-Adame, E. Maciá, B. Méndez, C. L. Roy and A. Khan, Semicond. Sci. Technol. 10, 797 (1995).
[12] F. Domínguez-Adame and E. Maciá, Semicond. Sci. Technol. 11, 1041 (1996).

[13] M. Kohmoto, L. P. Kadanoff, and C. Tang, Phys. Rev. Lett. 50, 1870 (1983).

[14] E. Maciá and F. Domínguez-Adame, Phys. Rev. Lett. 76, 2597 (1996).

[15] E. Diez, F. Domínguez-Adame, E. Maciá, and A. Sáchez, Phys. Rev. B (in press).

[16] K. Leo, P. Haring, F. Brüggemann, R. Schwedler, and K. Köhler, Solid State Commun. 84, 943 (1992).

[17] J. Feldmann, K. Leo, J. Shah, D. A. B. Miller, J. E. Cunningham, T. Meier, G. von Plessen, A. Schulze, P. Thomas, and S. Schmitt-Rink, Phys. Rev. B 46, 7252 (1992).

[18] N. G. Sun, D. Yuan, and W. D. Deering, Phys. Rev. B 51, 4641 (1995). 